\title{
Pulse Counting Sensorless Detection of the Shaft Speed and Position of DC Motor Based Electromechanical Actuators
}

\author{
Antonio Testa ${ }^{*}$, Salvatore De Caro ${ }^{\dagger}$, Tommaso Scimone*, and Romeo Letor ${ }^{* *}$ \\ ${ }^{* \dagger}$ Dept. of Electronic Engineering, Industrial Chemistry and Engineering, University of Messina, Messina, Italy \\ ${ }^{* *}$ STMicroelectronics, Catania, Italy
}

\begin{abstract}
Some of DC actuators used in home automation, office automation, medical equipment and automotive systems require a position sensor. In low power applications, the introduction of such a transducer remarkably increases the whole system cost, which justifies the development of sensorless position estimation techniques. The well-known AC motor drive sensorless techniques exploiting the fundamental component of the back electromotive force cannot be used on DC motor drives. In addition, the sophisticated approaches based on current or voltage signal injection cannot be used. Therefore, an effective and inexpensive sensorless position estimation technique suitable for DC motors is presented in this paper. This technique exploits the periodic pulses of the armature current caused by commutation. It is based on a simple pulse counting algorithm, suitable for coping with the rather large variability of the pulse frequency and it leads to the realization of a sensorless position control system for low cost, medium performance systems, like those in the field of automotive applications.
\end{abstract}

Key words: Automotive, DC actuators, Sensorless position control

\section{INTRODUCTION}

An ever increasing number of low power electromechanical actuators are being introduced into modern cars in order to improve comfort and safety. These actuators are generally equipped with Permanent Magnet DC motors, due to their low cost and quite mature technology. Although the ones used for driving fans and pumps are generally open loop operated, those used in window lifts, seat and mirror adjustments and air conditioning flap adjustments, require a quite precise closed loop position control. As an example, a DC electric actuator for power window applications encompasses two Hall Effect sensors, as shown in Fig.1. A two Hall Effect sensors approach is required to determine the position of the window from the rotor angular position and the direction of the motion. The introduction of these Hall Effect sensors and of the related additional wires, connectors

Manuscript received Jun. 3, 2013; accepted Mar. 17, 2014

Recommended for publication by Associate Editor Sanjib K. Panda.

†Corresponding Author: sdecaro@unime.it

Tel: +39-90-3977557, University of Messina

*Dept. of Electronic Engineering, Industrial Chemistry and Engineering,

University of Messina, Italy

${ }^{* *}$ STMicroelectronics, Italy and electronic circuits, remarkably increases the complexity of the system, and the extra cost may easily exceed one third of the cost of the whole actuator.

Low cost is mandatory for very large scale production devices, such as the DC actuators used in automotive applications. Although cost containment can be achieved with advanced design techniques, [3]-[5], a straightforward approach deals with the elimination of the position sensors. This can only be accomplished if an effective way to estimate the rotor position from the measurement of the motor electrical variables is provided. In a DC motor the shaft angular speed, and therefore the angular position, can be obtained from the actual values of the back Electromotive Force (EMF) and the armature current, through the mathematical model of the machine. However, even small errors in identifying the motor parameters, the parametric spread on machines belonging to the same production batch, or simply variations of the armature resistance due to temperature, lead to poor results in terms of position estimation precision.

An effective sensorless position estimation can be accomplished by taking into account the typical ripple of the 


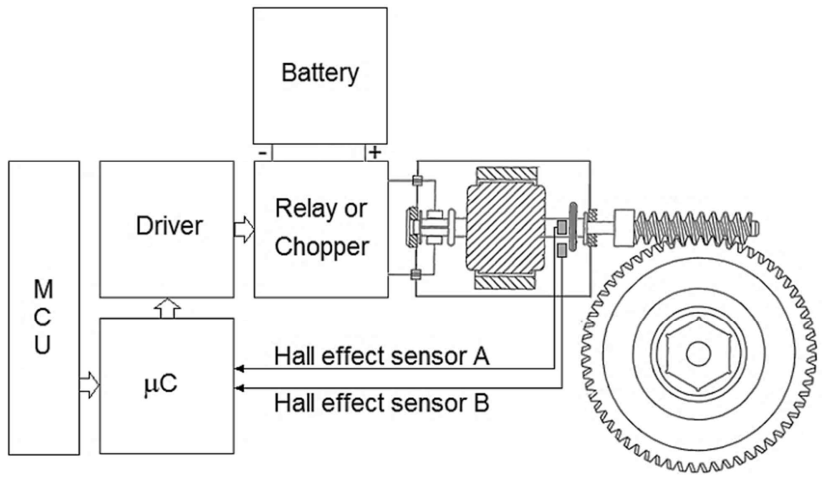

Fig. 1. A schematic of an electric power window actuator.

armature current caused in a DC machine by the commutation mechanism. In fact, since a given number of armature current pulses per round are generated, an incremental angular position measurement fully unaffected by motor parameter variations can be accomplished by counting the commutation pulses. Moreover, the detection of armature current pulses only requires a suitable current sensor, which is normally present on automotive DC actuators for control and safety reasons. However, in practice, the large variability of the pulse frequency and a strong sensitivity to brush bounces result in the detection of apparent, or false, pulses while true ones are often skipped. This makes such an approach very difficult to successfully implement, due to the incremental nature of the accomplished position estimation. Moreover, the shape of the commutation pulses may show differences even in machines of the same production batch, and it may also change along the operative life of a given DC motor. Therefore, the practical implementations of pulse counting position estimators generally do not comply with the precision, reliability and accuracy requirements of the above mentioned automotive applications. Moreover, they often require special converter topologies, additional power supplies or quite powerful microcontrollers.

The main drawbacks of armature pulse counting are resolved in this paper through an original approach based on a tunable band pass filter and a pulse count check and correction procedure. A tunable band pass filter, has been developed which is able to continuously adapt its center band according to variations of the motor speed in order to effectively eliminate from the armature current signal the DC component, switching harmonics and possible disturbances caused by brush bounces, windings non idealities, mechanical frictions and distortions of the excitation flux. As a result, quite clean signal at a frequency proportional to the rotor speed is obtained. Moreover, an active pulse count check and correction procedure has been implemented based on an accurate prediction of the pulse frequency, providing the means to compensate for the possible loss of pulses, as well as the improper detection of apparent pulses. According to experimental evidence, by exploiting the proposed approach it is possible to eliminate the Hall Effect position sensors in electric window lifters and in automotive DC actuators in general.

\section{SENSORLESS SPEED AND POSITION ESTIMATION IN DC MACHINES}

Sensorless speed and position estimation on vectorial AC motors and DC brushless drives has been extensively studied over the past four decades and a large number of papers have been published [6]-[12]. On the other hand, only few publications can be found in the literature dealing with sensorless position estimation techniques for brushed DC motors, [13]-[15]. In fact, unlike vectorial AC and Brushless DC motor drives, a position sensor is not required to control the output torque of brushed DC motor drives, as the commutation always holds orthogonal the field and armature flux densities. Moreover, DC motor drives can be very easily open loop driven, since the shaft speed is proportional to the back electromotive force, which in turn, at least at a sufficient speed level, can be approximated to the armature voltage. Therefore, position and speed sensors are present only in high performance DC drives, such as those used in robotics and factory automation applications. In these cases, a closed loop position control is required to reach the expected precision and dynamic performances. The precision offered by open loop speed operation is insufficient in automotive actuators, either for control purposes or to implement safety functions, such as the pinch protection in electrically powered window lifters. On the other hand, the low cost requirement strongly encourages the elimination of position sensors. As a result, the exploitation of sensorless control strategies represents a good compromise between performance and cost in these systems.

A simple way to estimate the shaft angular speed, and therefore the angular position, starting from measurements of the armature voltage $v_{a}$ and armature current $i_{a}$, is by exploiting the following equations carried out from the DC machine mathematical model:

$$
\begin{aligned}
& \omega_{r}=\frac{v_{a}-R_{a} i_{a}}{p p K_{e} \Phi_{e}} \\
& \alpha_{r}=\alpha_{r 0}+\int_{0}^{t} \omega_{r} d t
\end{aligned}
$$

where $\alpha_{r}$ and $\omega_{r}$ are the rotor position and rotor speed respectively, $\phi_{e}$ is the excitation flux, $R_{a}$ and $L_{a}$ are the armature resistance and armature inductance respectively, $p p$ is the pole pairs and $K_{e}$ is the machine constant.

However, since estimation of the rotor speed according to eq. (1) is quite inaccurate and sensitive to motor parameter variations, it is generally unsuitable for the considered applications. Some other sensorless techniques have been 


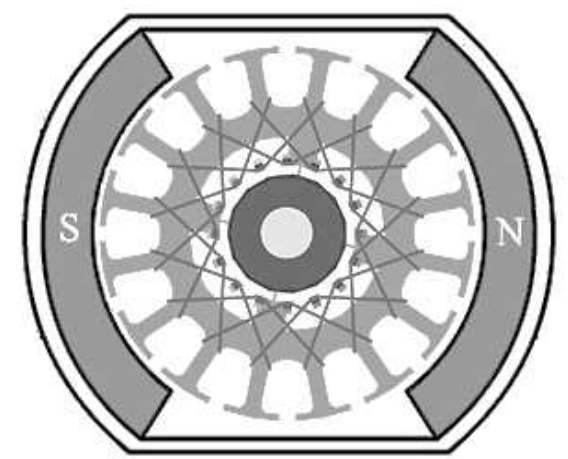

Fig. 2. Structure of a permanent magnet DC motor.

proposed in the past by exploiting state observers for estimating the speed and disturbance torque [16]. While effective, at least in the steady state, these techniques are too complex to be implemented on the low cost processors commonly used in automotive applications.

Another alternative for obtaining a precise sensorless position estimation is to take into account the typical ripple of the armature current caused in a DC machine by the commutation mechanism and rotor structure anisotropy.

DC motors for low power applications feature the structure shown in Fig. 2. While excitation is generally provided by permanent magnets [17], [18], the armature windings are made by coils wound around the armature teeth. Since two-layer windings are generally used, each armature slot hosts two sides of two different coils. Therefore, the number of coils is equal to the number of slots. The coil pitch is equal to one pole pitch and each coil encompasses some tens of turns.

Each winding encompasses $Z$ conductors where:

$$
Z=2 N_{s} N_{c}
$$

where: $N_{S}$ are the coils and $N_{\mathrm{C}}$ are the turns per coil.

Therefore, the total electromotive force induced on the conductors placed in slot $i$ is:

$$
e_{i}(t)=\frac{Z}{a N_{s}} e_{a i}(t)
$$

where: $a$ is the number of parallel paths between the brushes, and $e_{c i}(t)$ is the voltage induced on a single wire hosted in slot $i$. The total no load back EMF is given by:

$$
e_{a}(t)=\sum_{i=1}^{N_{S P}} e_{i}(t)
$$

where: $N_{S P}$ is the number of slots per pole.

As shown in Fig. 3, the back EMF at no load shows a periodical pulse every $\varepsilon=2 \pi / N_{S}$, as a result of the coil shifting. Therefore, $N_{S}$ pulses per round are produced and an additional back EMF AC component is generated which features an angular frequency $\omega_{p}$, which is equal to the rotor

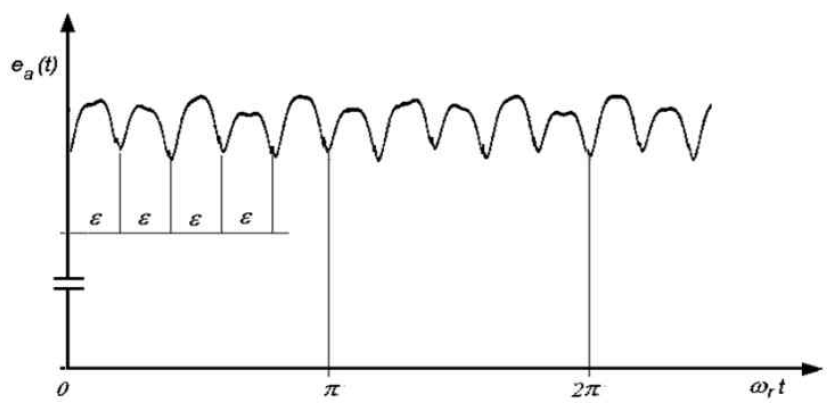

Fig. 3. Typical waveform of the no load back EMF $e a(t)$.

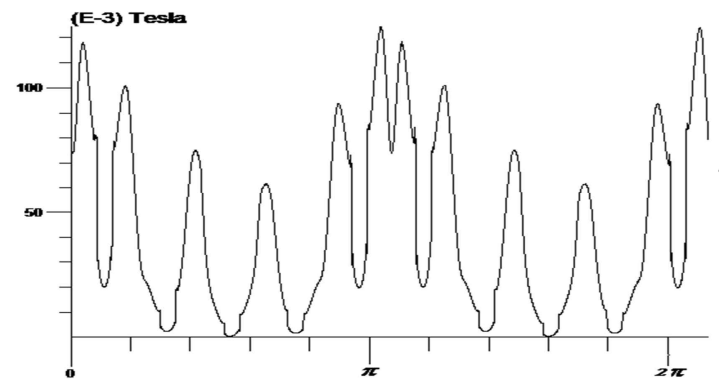

Fig. 4. Airgap excitation flux density distribution.

angular speed $\omega_{r}$ times the number of slots $N_{S}[19]$, [20].

Even if the excitation magnetomotive force is constant, the excitation flux in a DC machine shows a periodic variation. This is shown in Fig. 4, where it is possible to observe that the spatial distribution of the excitation flux density is composed of a mean DC component and two different AC components. The first $\mathrm{AC}$ component is generated from the saturation of the magnetic core induced by the excitation field and features an angular frequency that is twice $\omega_{r}$. The second AC component is produced by the saliency of the rotor core and features an angular frequency that is equal to $\omega_{p}$. This generates a further armature voltage ripple component at the same frequency as that caused by the commutation. The two components of the ripple at $\omega_{p}$ are almost in phase between them [21], [22].

Assuming that the ripple $\Delta e_{a}$ is a pure sinusoidal component of the total back EMF $e_{a}$, the mathematical model of a permanent magnet $\mathrm{DC}$ motor gives:

$$
\begin{aligned}
v_{a} & =R_{a} i_{a}+L_{a} \frac{d i_{a}}{d t}+e_{a}+\Delta e_{a} \\
e_{a} & =p p K_{e} \omega_{r} \Phi_{p m} \\
\Delta e_{a} & =p p K_{e} \omega_{r} K \Phi_{p m} \dot{s}\left(N_{s} \omega_{r} t\right) \\
T_{e} & =p p K_{e} \phi_{p m} i_{a} \\
\frac{d \omega_{r}}{d t} & =\frac{1}{J}\left(T_{e}-T_{l}\right)-F \omega_{r}
\end{aligned}
$$

where: $K$ is a constant that depends on the amplitude of the oscillation of the back EMF, $\phi_{p m}$ is the permanent magnet flux, $T_{e}$ and $T_{l}$ are the electromagnetic and load torques respectively, 


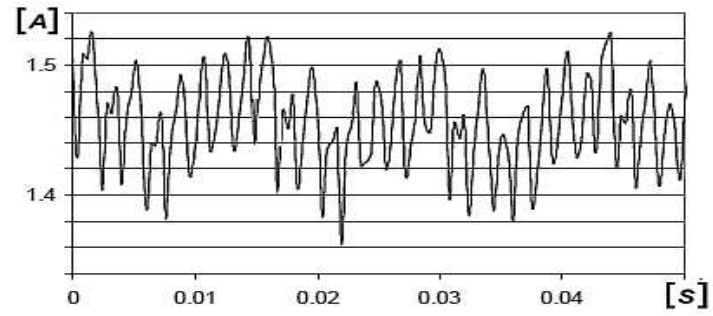

Fig. 6. Armature current ripple at steady state.

and $\mathrm{J}$ and $\mathrm{F}$ are the inertia and friction coefficients respectively.

The armature current $i_{a}$ can then be computed as:

$$
R_{a} i_{a}+L_{a} \frac{d i_{a}}{d t}=v_{a}-p p K_{e} \omega_{r} \Phi_{p m}\left[1+K \dot{s i n}\left(N_{s} \omega_{r} t\right)\right]
$$

By solving equation11, it turns out that:

$$
i_{a}=i_{a D C}+i_{a A C}+i_{\text {atrans }}
$$

where $i_{a D C}, i_{a A C}$ and $i_{\text {atrans }}$ are the mean, the ripple and the transitory components of the armature current $i_{a}$ respectively. They are given by:

$$
\begin{gathered}
i_{a D C}=\frac{v_{a}-p p K_{e} \omega_{r} \Phi_{p m}}{R_{a}} \\
i_{a A C}=\frac{p p K_{e} \omega_{r} K \Phi_{p m}}{R_{a}^{2}+L_{a}^{2} N_{s}^{2} \omega_{r}^{2}}\left[R_{a} \dot{s i n}\left(N_{s} \omega_{r} t\right)-L_{a} N_{s} \omega_{r} \cos \left(N_{s} \omega_{r} t\right)\right] \\
i_{\text {atrans }}=-\frac{v_{a}-p p K_{e} \omega_{r} \Phi_{p m}}{R_{a}} e^{-\frac{R_{a}}{L_{a}} t}
\end{gathered}
$$

As confirmed by the steady state experimental test shown in Fig. 6, the back EMF oscillation causes a ripple in the armature current featuring an angular frequency that is equal to the rotor angular speed, times the number of slots, as predicted by eq. (14). However, the current ripple waveform of Fig. 6 is not sinusoidal, since a remarkable distortion is caused in practice by winding asymmetries, armature reaction and magnetic core saturation. Moreover, disturbances are caused in practice by PWM switching, brush bounces, and mechanical phenomena [23]. Finally, the amplitude of the ripple changes with the amplitude of the mean component of $i_{a}$.

\section{THE PROPOSED SENSORLESS TECHNIQUE}

According to Eq. 14 and Fig. 6, the amount of current pulses per round generated by the commutation is related to the number of rotor slots. Therefore, an incremental position estimation technique has been developed according to the scheme of Fig. 7. The sensorless angular position estimation starts from the measurement of the armature current. Inexpensive current sensors, such as: shunt resistors, inductances or current mirrors can be successfully used. This is due to the fact that the frequency of the pulses, and not the amplitude of the armature current, is taken into account to

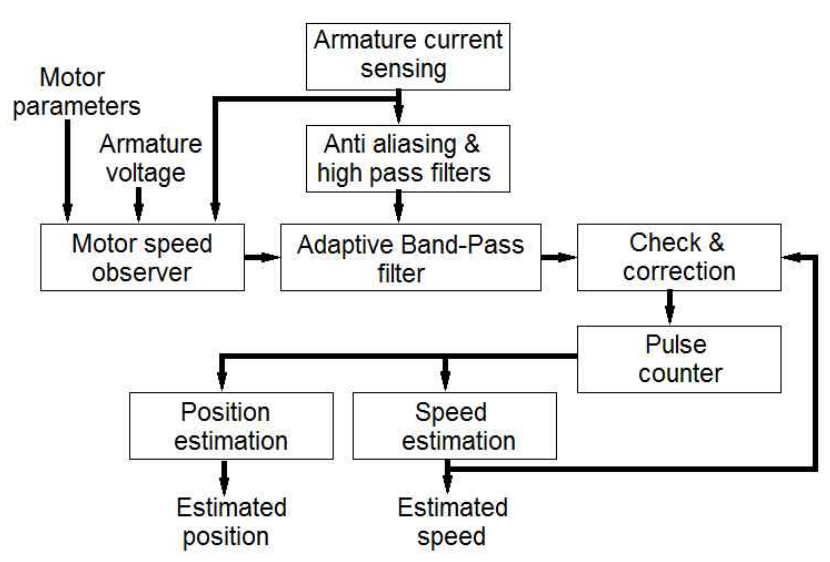

Fig. 7. The proposed sensorless technique.

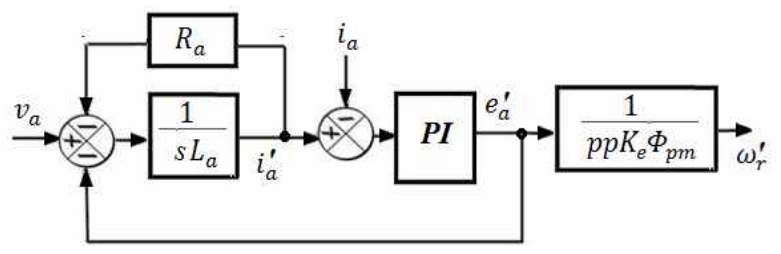

Fig. 8. The speed estimator.

TABLE I

Technical Data of a DC Electric Power Window Actuator

\begin{tabular}{|c|c|}
\hline Rated voltage & $12 \mathrm{~V}$ \\
\hline Rated output power & $50 \mathrm{~W}$ \\
\hline Stall torque & $.14 \mathrm{Nm}$ \\
\hline Rated speed & $3600 \mathrm{rpm}$ \\
\hline No load speed & $5400 \mathrm{rpm}$ \\
\hline Rated current & $8.5 \mathrm{~A}$ \\
\hline No load current & $\leq 3 \mathrm{~A}$ \\
\hline Stall current & $\leq 24 \mathrm{~A}$ \\
\hline Number of rotor slots & 10 \\
\hline Reduction gear ratio & $76: 1$ \\
\hline
\end{tabular}

estimate the angular position in the proposed approach. The combination of a high pass filter and an anti-aliasing filter featuring a $2 \mathrm{kHz}$ bandwidth then processes the armature current signal to eliminate the DC component as well as the high frequency switching harmonics, electromagnetic noise, and the effects of brush bouncing. A further band pass filter then isolates the harmonic component of the armature current at the angular frequency $\omega_{p}$. However, this is equal to the rotor angular speed $\omega_{r}$ times the number of slots $N_{S}$. As shown in Table I, where typical technical specifications of a DC electric power window actuator are given, the rated motor speed is $3600 \mathrm{rpm}$ and the number of rotor slots is ten. Therefore, in normal operation, the frequency of the armature current ripple ranges from 0 to $600 \mathrm{~Hz}$. Such a large variability in the frequency prevents the use of a conventional band pass filter and leads to the introduction of a tunable band pass filter. Therefore, a switched capacitor filter has been selected, whose center band is continuously adjusted through a clock 


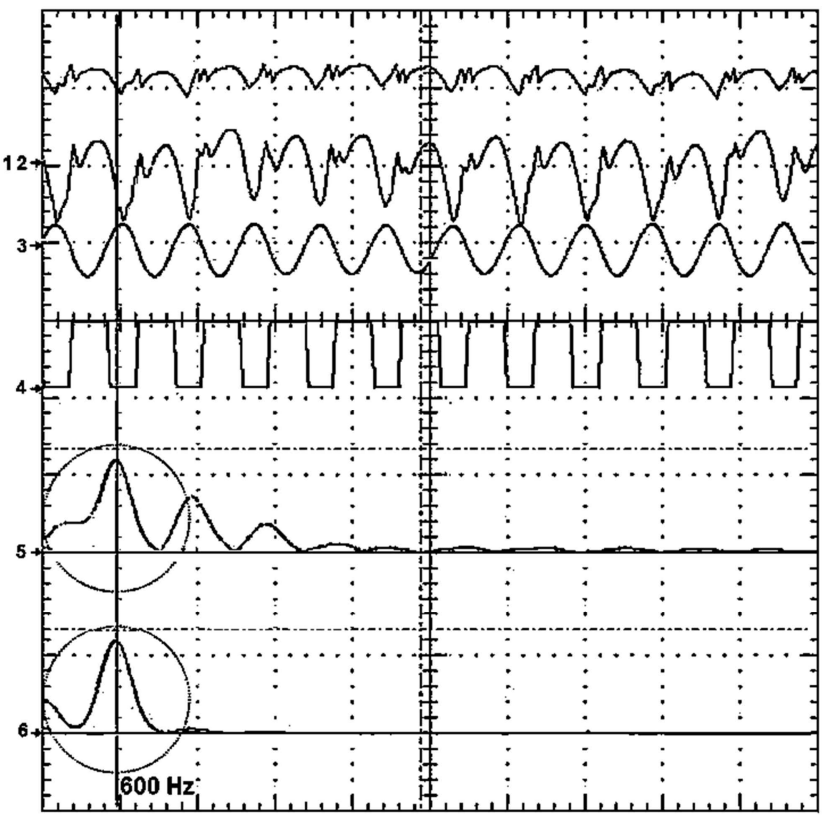

Fig. 9. Steady state test: 1) $i_{a}[5 \mathrm{~A} / \mathrm{div}]$; 2) signal at the output of the high-pass filter [500mV/div]; 3) signal at the output of the adaptive pass-band filter[500m V/div]; 4) squared signal ready to be processed[5V/div];Time [2 $\mathrm{ms} / \mathrm{div}]$; 5) FFT of the signal at the output of the high-pass filter, Frequency [625 Hz/div]; 6) FFT of the signal at the output of the adaptive pass band, Frequency [625 Hz/div].

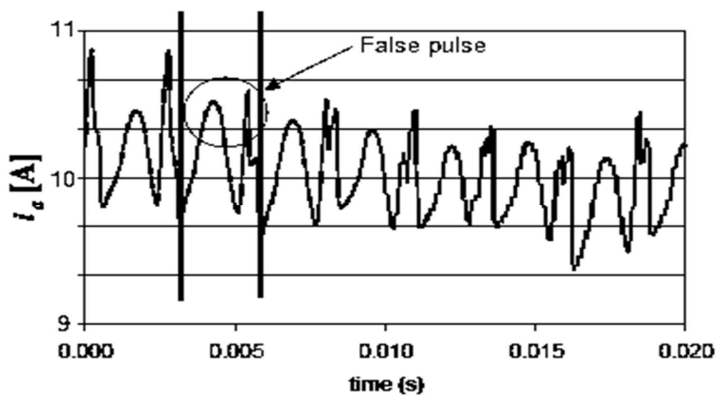

Fig. 10. False pulse occurring at heavy load.

signal in order to follow the variations of the frequency of $i_{a A C}$ The frequency of the clock signal is in turn determined by a speed estimation $\omega_{r}$ ' obtained through a closed loop speed observer based on eq. (1), as shown in Fig. 8. However, high accuracy in estimating the rotor speed is not required in this case, since $\omega_{r}$ ' is only used to drive the allowed band of the filter in the frequency window in which $i_{a A C}$ is expected to be located.

The signals measured in different points of the scheme in Fig. 7 during a steady state test are shown in Fig. 9. The output signal of the adaptive band pass filter only contains the first harmonic component of the current ripple $(600 \mathrm{~Hz})$, since all of the other harmonics have been properly filtered. A peak detector is then tasked to detect the armature current pulses. However, due to electromagnetic noise, motor structure imperfections, mechanical vibrations, variable friction and

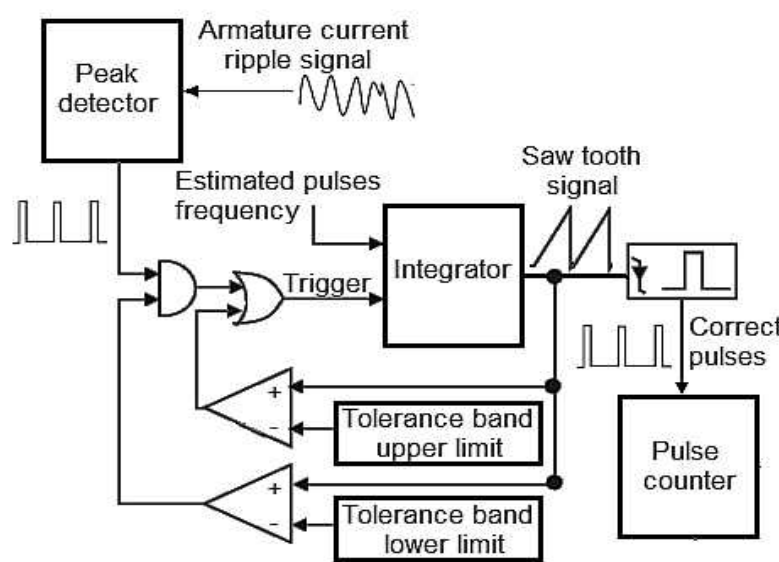

Fig. 11. Check and correction procedure.

unfiltered residual harmonic components, a simple peak detector does not ensure the requested level of reliability in estimating the rotor position, since some pulses may be missed, or false pulses may be taken into account as shown in Fig. 10. This is a big problem for an incremental position estimator, since errors may accumulate unacceptably.

A pulse check and correction procedure has been developed as shown in Fig. 11. The actual value of the frequency of the armature current pulses $f_{\text {pest }}(t)$ is first computed starting from the estimated motor speed $\omega_{\text {rest }}$ and the derivative of the rotor speed estimation $\omega_{r}$ ' provided by the observer of Fig. 8 as:

$$
f_{\text {pest }}(t)=2 \pi N_{S}\left(\omega_{\text {rest }}+t \frac{d \omega_{r}^{\prime}}{d t}\right)
$$

where: $t_{0} \leq t \leq t_{f}$, and $t_{0}$ is the instant in which the last pulse has been detected and $t_{f}$ is the instant in which the next pulse will be detected. On line adjustment of $f_{\text {pest }}(t)$ by exploitation of the speed observer is essential when coping with special operating conditions such as a motor starts or during externally forced stops.

The estimated ripple frequency is then integrated in order to obtain a saw tooth signal triggered by the detection of the last pulse. Ideally, when a new pulse is detected, the result of the integration is one. However, the frequency estimation is fairly affected by errors and may show a small ripple. Therefore, a suitable tolerance band is defined to check for the correctness of pulses identified by the peak detector.

More specifically, as shown in Fig. 12, if a new pulse is detected, the integration of the estimated frequency reaches a value falling in the tolerance band, the pulse is identified as a true one and the integrator is reinitialized. On the other hand, if the output of the integrator falls out of the tolerance band, a false pulse is detected and the integration is continued. Moreover, as shown in Fig. 13, if the output of the integrator exceeds the upper limit of the tolerance band, a missed pulse is identified and the integrator is reinitialized. A suitable pulse 


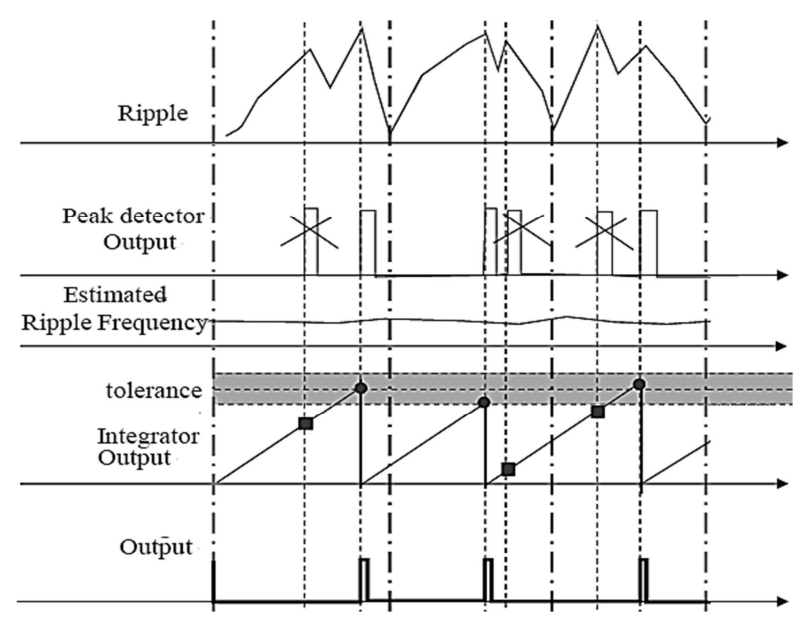

Fig. 12. False pulse detection and correction.

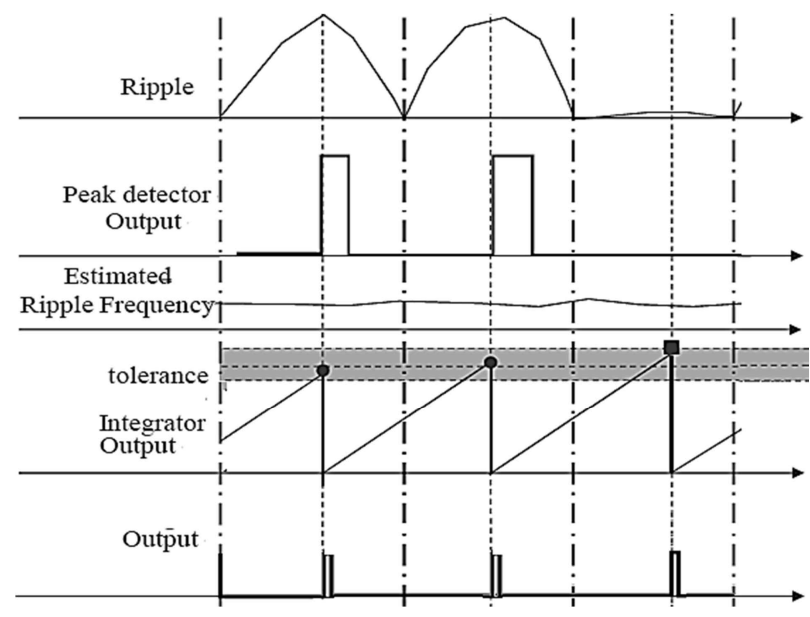

Fig. 13. Missed pulse detection and correction.

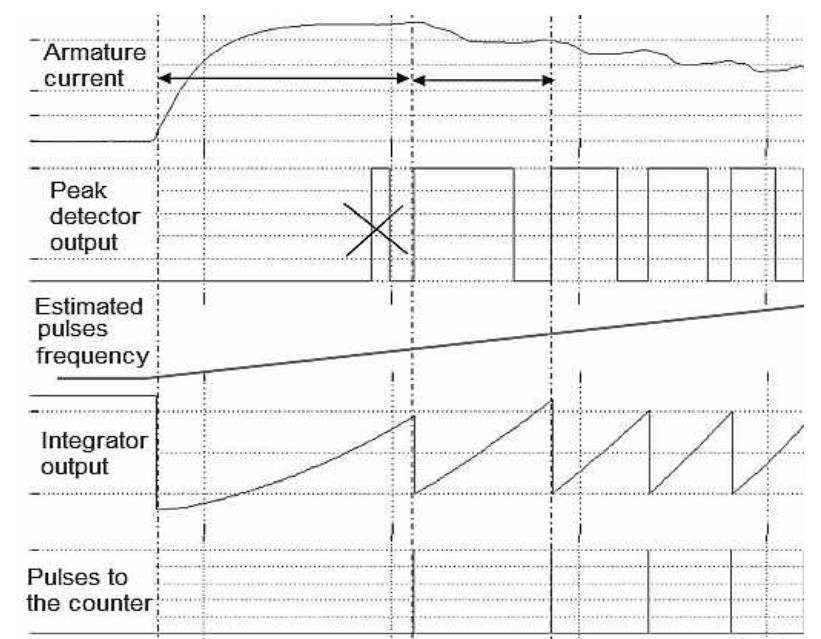

Fig. 14. False pulse detection: current [1.5A/div]; frequency $[35 \mathrm{~Hz} / d i v]$; time [.1s/div].

generator then sends a pulse to the counter whenever the integrator is reinitialized. Simulations are presented in Fig. 14 and 15 dealing with the detection of false pulses and missed

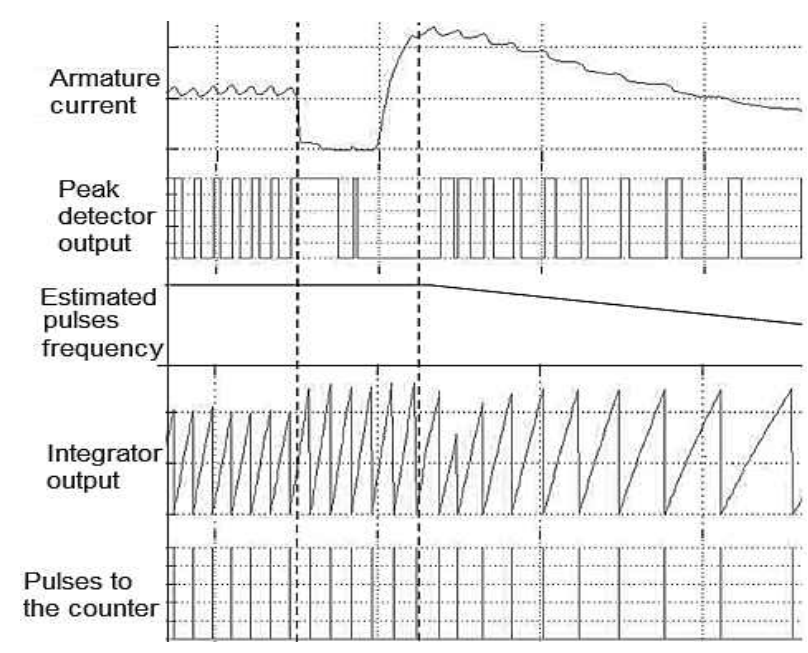

Fig. 15. Missed pulse detection: current [1.5A/div]; frequency $[100 \mathrm{~Hz} / \mathrm{div}] ;$ time $[.05 \mathrm{~s} / \mathrm{div}]$.

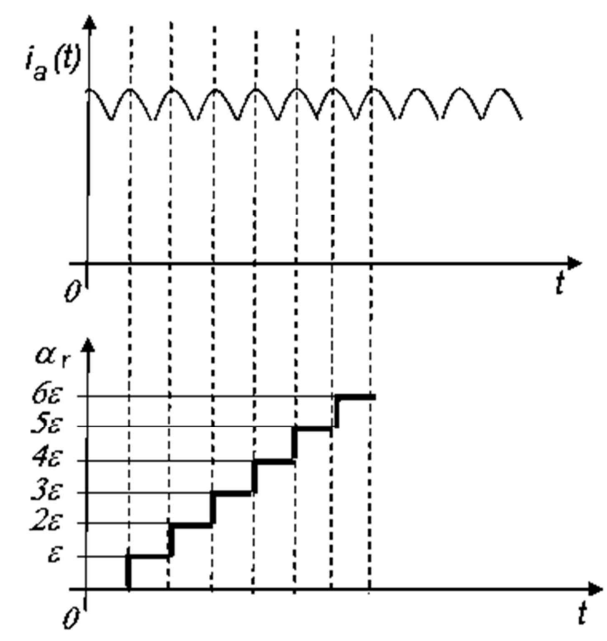

Fig. 16. Rotor position computation.

pulses respectively.

As shown in Fig. 16, the rotor position is finally estimated by counting the armature current pulses according to:

$$
\alpha_{r}=N \varepsilon
$$

where $\alpha_{r}$ is the mechanical position of the rotor shaft and $N$ is the number of detected pulses.

Moreover, the angular speed $\omega_{\text {rest }}$ of the rotor is also computed as:

$$
\omega_{\text {rest }}=\frac{N_{T}}{T} \varepsilon
$$

\section{EXPERIMENTAL EVALUATIONS}

The proposed sensorless technique has been implemented on a development board, tailored around a custom multi-island Intelligent Power Module (IPM), as shown in Fig. 17.

The IPM encompasses: 


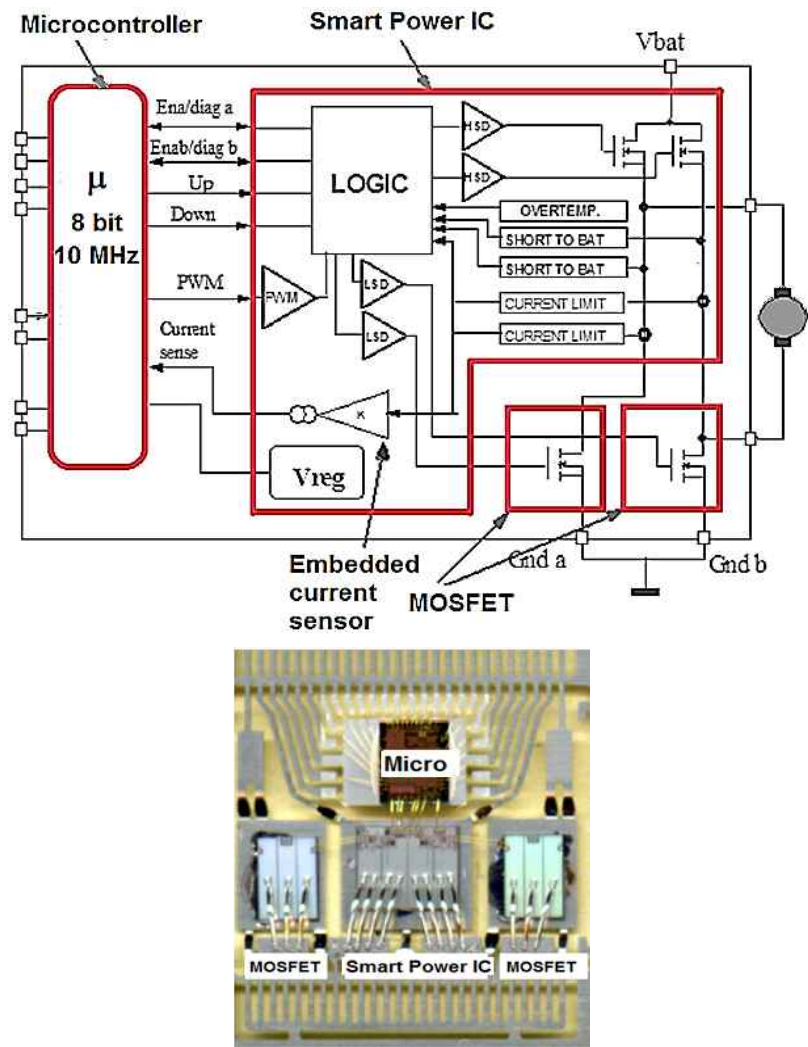

Fig. 17. Schematic of the IPM used in the experimental system (up) and a picture of the IPM (down).

- a monolithic smart power device, including the high side switches of an $\mathrm{H}$ bridge chopper, logic, protections and output current sensing circuits;

- $\quad$ the two low side MOSFETs of the chopper;

- a low cost $10 \mathrm{MHz}, 8$-bit microcontroller.

The board also includes a monolithic switched capacitor filter.

First, no load tests were accomplished by taking out the DC motor from the actuator assembly. Fig. 17 deals with a motor start, followed by an externally forced stop. A cumulative error lower than $0.4 \%$ has been recorded in estimating the rotor position.

Then, some tests were accomplished on a real medium segment car window lifter.

Figs. 19, 20 and 21 deal with a full window lift test. The armature current drops just after the motor start due to the tensioning of the steel cable driving the window through a pulley. The armature current then rises up to the rated value and the motor runs at a roughly constant speed until the window reaches the end of the rail. At this point the armature current increases rapidly, due to the mechanical lock. This continues until the pinch protection turns the motor off.

The window position during a full lift when it is left free to move along its rail is shown in Fig. 22. Reconstruction of the window position starting from the estimated motor angular position is compared with that obtained exploiting a $2048 \mathrm{ppr}$
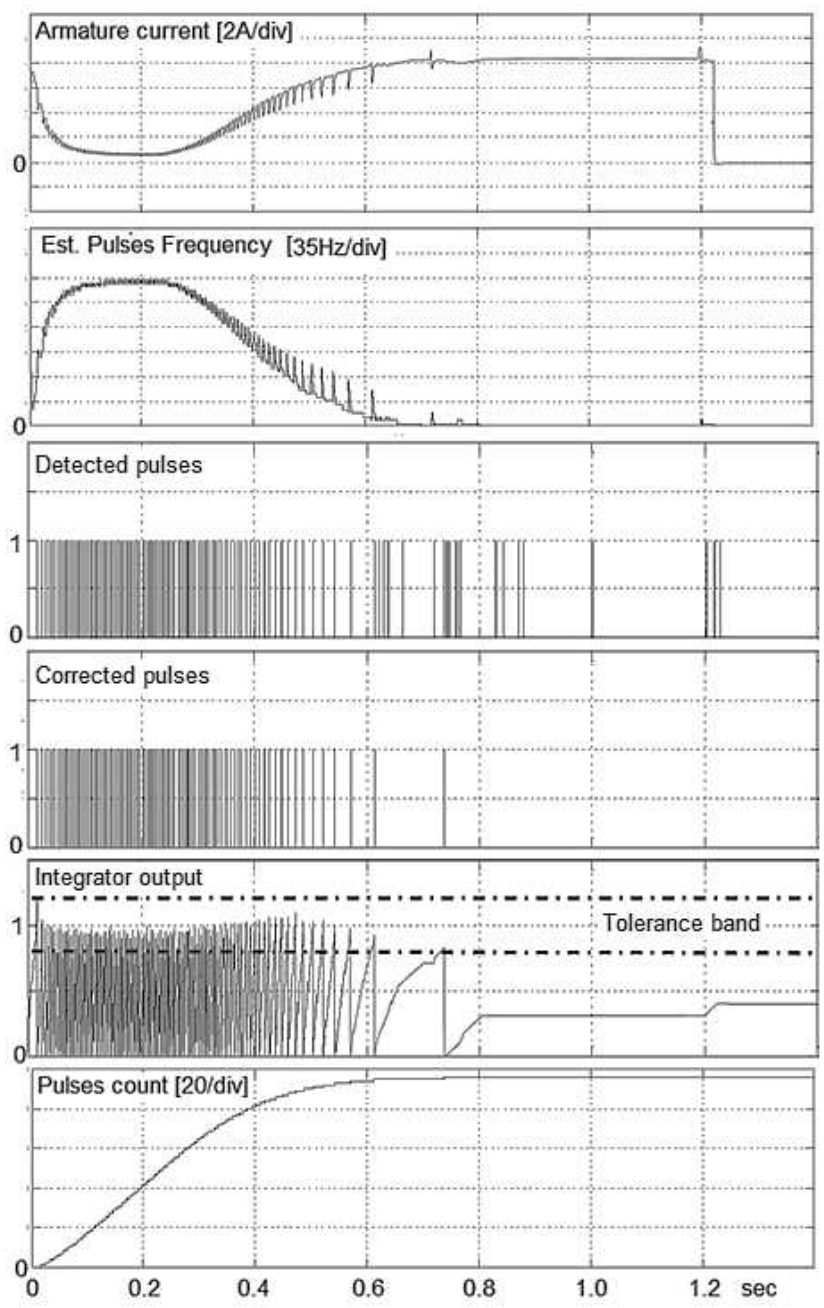

Fig. 18. No load test: motor start and forced stop.

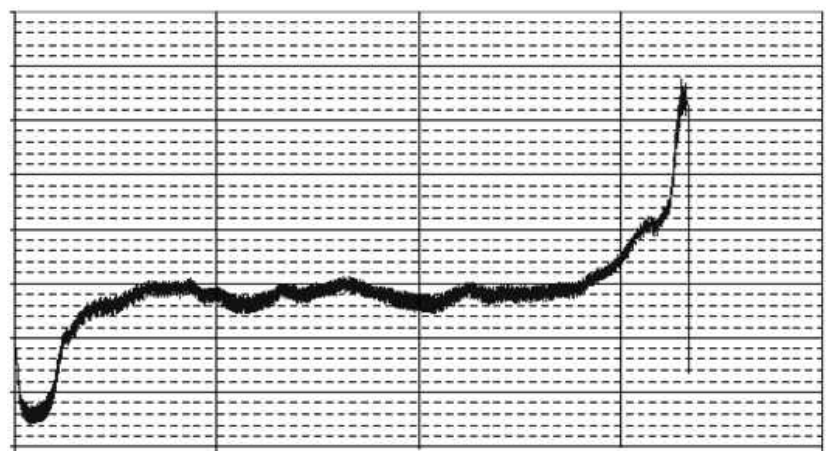

Fig. 19. Full window lift: $i_{a}[2 A / d i v]$; time [ $\left.5 s / d i v\right]$.

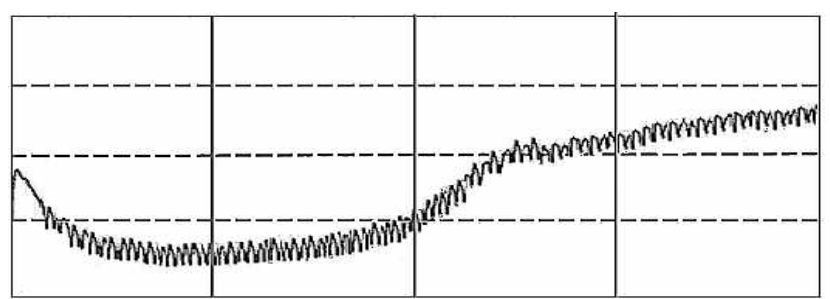

Fig. 20. Full window lift - Start: $i_{a}[1 \mathrm{~A} / \mathrm{div}]$; time [.5s/div]. 


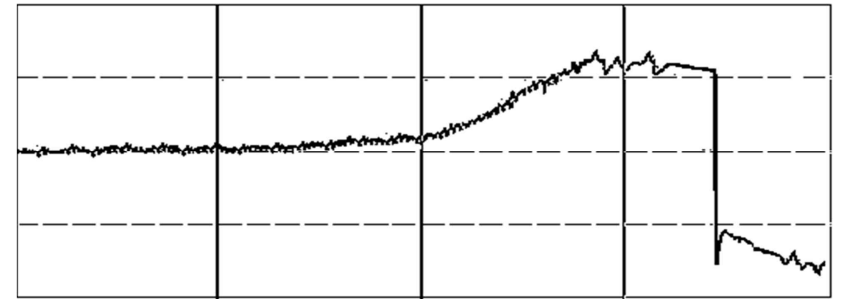

Fig. 21. Full window lift - Stop: $i_{a} \mathrm{t}[4 A / d i v]$; time [.35s/div].

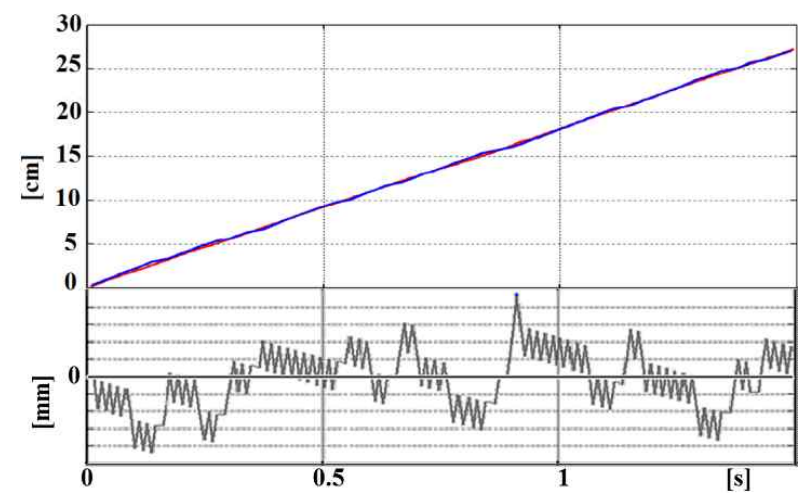

Fig. 22. Window free to move test. Up: measured (red) and estimated (blue) position . Down: error [ $1 \mathrm{~mm} / \mathrm{div}]$.

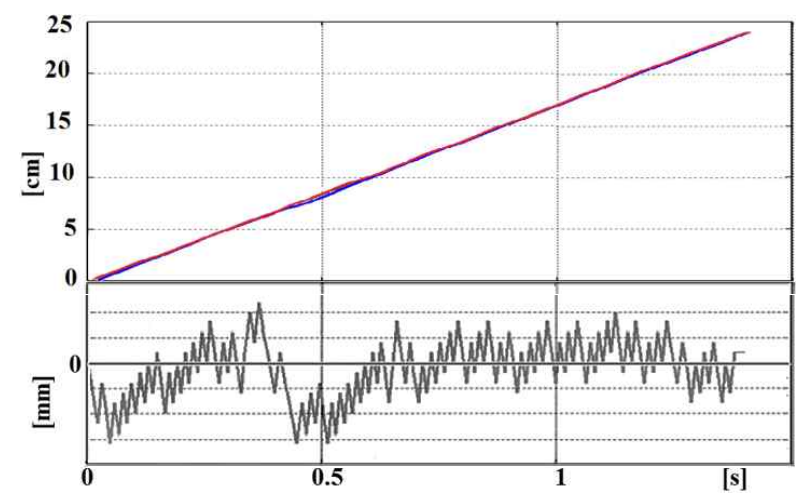

Fig. 23. Constant load test.Up: measured (red) and estimated (blue) position. Down: error [1 $1 \mathrm{~mm} / \mathrm{div}]$.

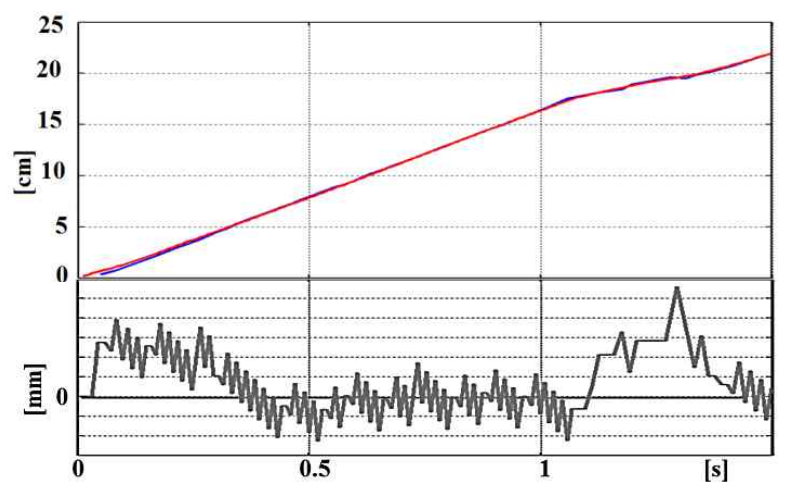

Fig. 24. Step load test. Up: measured (red) and estimated (blue) position [ $5 \mathrm{~cm} / \mathrm{div}]$. Down: error [ $1 \mathrm{~mm} / \mathrm{div}]$.

encoder. A maximum error that is lower than $4 \mathrm{~mm}$ and a cumulative final error that is lower than $2 \mathrm{~mm}$ are obtained. In

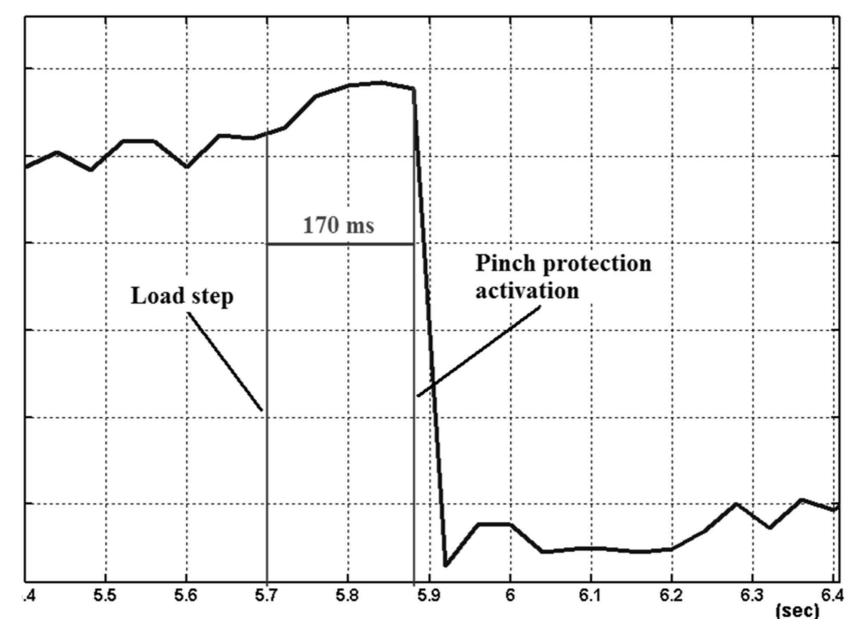

Fig. 25. Sensorless pinch protection activation: $i_{a \text {. }}[1 \mathrm{~A} / \mathrm{div}]$.

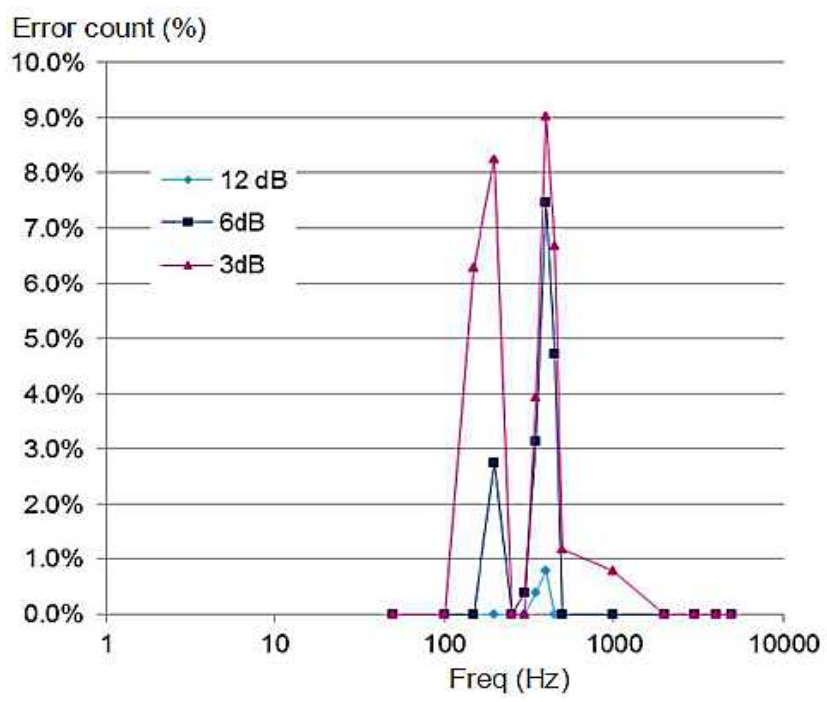

Fig. 26. Percent count errors occurring when a disturbance is added to the measured armature current signal.

Figs. 23 and 24, the same variables are shown when a constant resistive force and a step load force are applied to the window respectively. In both cases the system correctly computes the position of the window from the estimated angular position of the motor shaft, until the window reaches its final position.

All electric power window actuators must include an anti-pinch protection for safety reasons. Therefore, it is mandatory that the developed technique be able to provide an estimation of the rotor angular position suitable to properly activate the anti-pinch protection. In order to evaluate the consistency of the developed technique from this point of view, a pinch protection algorithm has been introduced into the scheme shown in Fig. 7. It is practically implemented by exploiting an 8 bit microcontroller for accomplishing the angular position estimation. Such an algorithm is based on a conventional obstacle detector composed of a low pass filter and a threshold comparator. 
When an obstacle is encountered, the filter output drops to under a predefined threshold and the pinch protection is activated which turns off the motor to stop the window. The response time and sensitivity of the pinch protection can be adjusted by adjusting the filter cutoff frequency and the threshold amplitude. In a conventional electric power window system, the anti-pinch protection is activated by an estimation of the window speed obtained from the output of Hall Effect sensors. In this case, the real window speed is computed according to equation 16. A test has been carried out where the window lift is open loop performed. However, a speed feedback signal is provided to the pinch protection. As shown in Fig. 25, when an obstacle is detected, the motor is turned off and the window promptly stops. The obtained response time (170 $m s$ ), is suitable for achieving a reasonable safety level.

In order to assess the robustness of the proposed technique a sinusoidal disturbance signal has been injected into the armature current sensor. Varying the frequency and the ratio between the amplitudes of the armature current ripple and the injected signal yields the results presented in Fig. 24. The largest percent error in pulse counting occurs when the frequency of the disturbance approaches that of the ripple. An error that is less than $9 \%$ is obtained, confirming the robustness of the developed technique.

\section{CONCLUSIONS}

The introduction of a position transducer in low power electromechanical DC actuators for automotive applications remarkably increases the whole system cost. On the other hand, open loop speed operations are unpractical. These facts have powered the development of sensorless angular position estimation techniques in order to obtain a good compromise between performance and cost. A well-known approach dealt with armature pulse counting. However some major problems must be resolved due to the large variability of the pulse frequency and the sensitivity to disturbances. An original pulse counting approach has been presented in this paper based on a tunable band pass filter and a pulse count check and correction procedure. According to experimental results the proposed technique makes possible the elimination of the Hall Effect position sensors in electric window lifter and in automotive DC actuators in general.

\section{REFERENCES}

[1] J. M. Miller, A. Emadi, A.V. Rajarathnam, and M. Ehsani, "Status and future trends in more electric car power systems," Vehicular Technology Conference, 1999 IEEE 49th, Vol. 2, pp. 1380-1384, 1999.
[2] A. Emadi, Handbook of Automotive Power Electronics and Motor Drives, CRC Taylor \& Francis, 2006.

[3] C. C. Chan and K. T. Chau, Modem Electric Vehicle Technology, Oxford University press, 2001.

[4] F. C. Lee, J. D. van Wyk, D. Boroyevich, G. Q. Lu, Z. Liang, and P. Barbosa, "Technology trends toward a system-in-a-module in power electronics," IEEE Circuits and Systems Magazine, Vol. 2, No.4, pp. 4-23, 2002.

[5] R. Letor, G. Di Caro, A. Russo, and N. Liporace, "Mixed system integration simplifies the design and the architecture of automotive power actuators," IEEE Industry Applications Annual Meeting, Vol. 2, pp. 1259-1263, 2003.

[6] L. Kreindler, A. Testa, and T. A. Lipo, "Position sensorless synchronous reluctance motor drive using the stator phase voltage third harmonic," in Proc. 1993 IEEE Industry Applications Society Annual Meeting, Vol. 1, pp. 679-686, 1993.

[7] A. Consoli, G. Scarcella, and A. Testa, "Industry application of zero-speed sensorless control techniques for PM synchronous motors," IEEE Trans. Ind. Appl., Vol. 37, No. 2, pp. 513-521, Mar./Apr. 2001,

[8] B. Fahimi, A. Emadi, and B. Sepe Jr., "Position sensorless control," IEEE Industry Applications Magazine, Vol. 10, No. 1, pp. 40-47. Jan./Feb. 2004,

[9] A. Testa, D. Triolo, A. Consoli, G. Scarcella, and G. Scelba, "Sensorless airgap flux position estimation by injection of orthogonal stationary signals," Proceedings of IEEE PESC '05, Power Electronics Specialists Conference, pp. $1567-1573,2005$.

[10] A. Kilthau and J. M. Pacas, "Sensorless control of the synchronous reluctance machine," Journal of Power Electronics, Vol. 2, No. 2, pp. 95-103, Apr. 2002.

[11] J. Holtz, "Developments in sensorless AC drive technology," International Conference on Power Electronics and Drives Systems (PEDS), pp. 9-16, 2005.

[12] K.-W. Lee and J.-I. Ha, "Evaluation of Back-EMF estimators for sensorless control of permanent magnet synchronous motors," Journal of Power Electronics, Vol. 12, No. 4, pp. 604-614, Jul. 2012.

[13] A. Consoli, S. De Caro, G. Bottiglieri, R. Letor, R. Ruggeri, and A. Testa, "Sensorless shaft position detection of low power DC motor drives," in Proc. Symposium on Power Electronics, Electrical Drives, Advanced Electrical Motors (SPEEDAM 2004), 2004

[14] G. Bottiglieri, A. Consoli, S. De Caro, R. Letor, R. Ruggeri, and A. Testa, "Sensorless position control of DC actuators for automotive applications," IEEE Industry Applications Conference, 2004. 39th IAS Annual Meeting, Vol. 2, pp. 1217-1224, 2004.

[15] A. Testa, S. De Caro, and R. Letor "Estimation of the shaft position on low-cost DC actuators," Proceedings of IEEE ISIE2010, International Symposium on Industrial Electronics, pp. 440-445, 2010.

[16] G. S. Buja, R. Menis, and I. Valla "Disturbance torque estimation in a sensorless DC drive," IEEE Trans. Ind. Electron., Vol. 42, No. 4, Pp. 351-357, Aug. 1995.

[17] T. Kenjo and S. Nagamori, Permanent-Magnet and Brushless DC Motors, Oxford, Clarendon Press, 1985.

[18] E. S. Hamdi, Design of Small Electric Machines, John Wiley \& Sons, 1994

[19] J. Cros, G. C. R. Sincero, and P. Viarouge "Design method for brush permanent magnet DC motors" IEEE International Electric Machines and Drives Conference (IEMDC' '09), pp. 1625-1632, 2009. 
[20] M. Wing, and J. F. Gieras " Calculation of the steady state performance of small commutator permanent magnet DC motors: classical and finite element approaches," IEEE Trans. Magn., Vol. 28, No. 5, pp. 2067-2071, Sep. 1992.

[21] K. Hameyer and R. J. M. Belmans "Permanent magnet excited brushed DC motors," IEEE Trans. Ind. Electron. Vol. 43, No. 2, pp. 247-255, Apr. 1996.

[22] M. K. Jamil and N. A. Demerdash, "Harmonics and core losses of permanent magnet DC motors controlled by chopper circuits," IEEE Trans. Energy Convers., Vol. 5, No. 2, pp. 408-414, Jun. 1990.

[23] M. Haji and H. A. Toliyat, "Rotor eccentricity fault detection of a DC motor," Industrial Electronics Society, IECON'01 The 27th Annual Conference of the IEEE, Vol. 1, pp. 591-596, Dec. 2001.

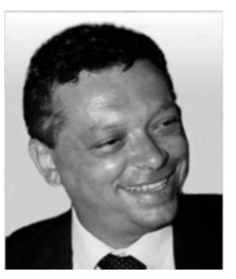

Antonio Testa (M'91) was born in Catania, Italy, in 1962. He received his degree in Electrical Engineering from the University of Catania, Catania, Italy, in 1988. From 1989 to 1990, he was a recipient of an SGS-Thomson Grant. From 1990 to 1998, he was an Assistant Professor of Electrical Drives and Power Electronics in the Department of Electrical, Electronic, and Systems Engineering, University of Catania. He is presently a Professor of Electric Machines and Power Electronics at the University of Messina, Messina, Italy. In 1991, he was a Visiting Researcher at the University of Wisconsin, Madison, WI, USA. His current research interests include the sensorless control of electrical drives, advanced digital control systems, power converters, power devices, and electromagnetic compatibility.

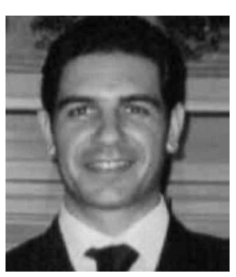

Salvatore De Caro (S'03-M'07) was born in Frankfurt, Germany. He received his M.S. degree in Electronic Engineering from the University of Messina, Messina, Italy, in 2000, and his Ph.D. degree in Electrical Engineering from the University of Catania, Catania, Italy, in 2004 . He is presently a Researcher at the University of Messina, where he is involved in the field of power electronics. His current research interests include power converters, power devices, generation systems from renewable energy sources, fuel cells and energy storage systems.

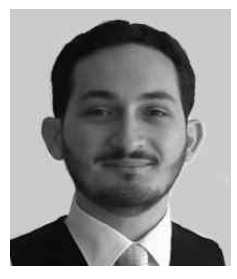

Tommaso Scimone was born in Messina, Italy, in 1981. He received his M.S. degree in Electronic Engineering from the University of Messina, Messina, Italy, in 2007. In 2008, he began pursuing his $\mathrm{PhD}$ degree in Energy at the Scuola Superiore of Catania, University of Catania, Catania, Italy. $\mathrm{He}$ is presently a Research Fellow in the Department of Electronic Engineering, Industrial Chemistry and Engineering, University of Messina. His research project is oriented toward the study of the accumulation devices equipping generation systems from renewable energy sources. His current research interests include high power $\mathrm{DC} / \mathrm{DC}$ and $\mathrm{DC} / \mathrm{AC}$ converters.

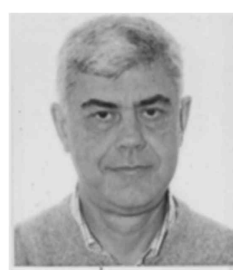

Romeo Letor received his degree in Electrical Engineering from the University of Naples, Naples, Italy, in 1985. After graduating, he took several roles at STMicroelectronics, Catania, Italy, where he has been working since 1987. He has many years of experiences in power electronics, power management, and automotive electronics. He has several patents and he has authored many papers for important worldwide conferences. He is currently leading the development of advanced projects in the STM automotive products group. 